\title{
Benefit-risk assessment of pitavastatin for the treatment of hypercholesterolemia in older patients
}

\author{
Kevin W Chamberlin ${ }^{1,2}$ \\ William L Baker ${ }^{1,2}$ \\ 'School of Pharmacy, the University \\ of Connecticut, Storrs, CT, USA; \\ ${ }^{2}$ School of Medicine, the University of \\ Connecticut, Farmington, CT, USA
}

This article was published in the following Dove Press journal:

Clinical Interventions in Aging

16 April 2015

Number of times this article has been viewed

REVIEW

\begin{abstract}
With the practice-shifting changes made with the most recent guidelines for treating blood cholesterol, more older patients may be prescribed statin therapy. Therefore, it is imperative that practitioners have not only a working knowledge of information related to statins, but more specifically to their efficacy and safety in elderly populations. Pitavastatin is the most recent statin to receive regulatory approval. It is indicated for the treatment of primary hyperlipidemia or mixed dyslipidemia as an adjunctive therapy to diet. The overall body of evidence for the efficacy and safety of pitavastatin in elderly patients is small. The available data suggest that the ability of pitavastatin to lower low-density lipoprotein cholesterol in elderly patients is at least similar, and may be greater than that seen in comparatively younger cohorts. Taken together, the limited available data suggest that pitavastatin is effective at improving lipid parameters in elderly patients with a similar safety profile to other agents in the class. Until data become available distinguishing pitavastatin from the other available options, its ultimate role in the hyperlipidemia treatment armamentarium remains unclear.
\end{abstract}

Keywords: pitavastatin, hypercholesterolemia, elderly

\section{Introduction to management of hypercholesterolemia in elderly patients}

It has been well established that elevated serum cholesterol levels are associated with increasing cardiovascular risk as the population ages. ${ }^{1-3}$ Meta-analyses have suggested significantly higher cardiovascular mortality rates in older individuals compared with their younger cohorts, with a 10-fold higher risk seen with every $38.6 \mathrm{mg} / \mathrm{dL}$ increase in total cholesterol (TC) seen. ${ }^{2}$ Trials with statins in older patients ( $>65$ years of age) with clinical atherosclerotic cardiovascular disease show significant reductions in total mortality (relative risk $0.78,95 \%$ confidence interval 0.65 to 0.89 ). ${ }^{4}$ Thus, prescribing cholesterol-lowering therapy in the form of statins to reduce cardiovascular risk in older patients is of considerable benefit. With the practice-shifting changes made with the most recent American College of Cardiology/American Heart Association guidelines for treating blood cholesterol, more elderly patients may be prescribed statin therapy. ${ }^{5}$ These guidelines expand the use of statins for primary prevention; a change that is most notable in older persons. Statins are recommended in patients who have 10 -year atherosclerotic cardiovascular disease risk of $\geq 7.5 \%{ }^{6}$ This means that most men over 60 years and women over 70 years of age will be eligible to receive statin therapy. ${ }^{5}$ Of concern to some is the safety of cholesterol-lowering drug therapy in the aging population; an issue likely to increase in prevalence with these expanded use guideline recommendations. This can be particularly troublesome with the development 
of frailty which increases patients' susceptibility to adverse events. ${ }^{1,6}$ Therefore, it is imperative that practitioners have not only a working knowledge of information related to statins, but more specifically to their efficacy and safety in older populations.

Pitavastatin (Livalo ${ }^{\circledR}$; Kowa Pharmaceuticals America, Montgomery, AL, USA) is the most recent statin to receive regulatory approval. It is indicated for the treatment of primary hyperlipidemia or mixed dyslipidemia as an adjunctive therapy to diet. ${ }^{7,8}$ The aim of this article is to review the pharmacologic, pharmacokinetic, efficacy, and safety data of pitavastatin specifically in older populations.

\section{Review of pharmacology, mode of action, and pharmacokinetics of pitavastatin in the elderly Pharmacology of pitavastatin}

Pitavastatin mimics other statins by inhibiting HMG-CoA (3-hydroxy-3-methylglutaryl-coenzyme A) reductase to reduce low-density lipoprotein cholesterol (LDL-C), elevated TC, Apo B, and triglycerides (TG) and to increase highdensity lipoprotein cholesterol (HDL-C). ${ }^{7}$ In contrast with other statins, pitavastatin contains a cyclopropyl group that fits within the hydrophobic areas of the HMG-CoA reductase enzyme, partially explaining its potent inhibitory activities. ${ }^{8}$ Studies have described pitavastatin to have a 2.4- to 6.8-fold greater potency than simvastatin and pravastatin, respectively. ${ }^{9}$ Further, pitavastatin inhibits cholesterol synthesis greater than simvastatin (2.9-fold) and atorvastatin (5.7-fold), and its inhibitory effects on sterol synthesis appear to be liver selective and significantly stronger than simvastatin..$^{10,11}$

\section{Pharmacokinetics of pitavastatin}

Pitavastatin is a potent and synthetic HMG-CoA reductase inhibitor, with a structure especially similar to fluvastatin and rosuvastatin. ${ }^{12}$ Comparison of pharmacokinetic attributes (Table 1) shows that pitavastatin has the highest bioavailability amongst all statins, and that it reaches peak plasma concentration $\left(\mathrm{C}_{\max }\right)$ within 1 hour after oral administration, the fastest of the seven approved statins in the United States. ${ }^{8,13}$ Administration of pitavastatin with a high-fat meal decreases the $\mathrm{C}_{\max }$ by $43 \%$; however, the area under the curve (AUC) is not significantly reduced. ${ }^{7}$ Further, the AUC of pitavastatin remains unchanged whether it is administered in the morning or evening.?

Pitavastatin is highly protein bound, more than $99 \%$, predominantly to albumin and alpha ${ }_{1}$-acid glycoprotein. ${ }^{7}$

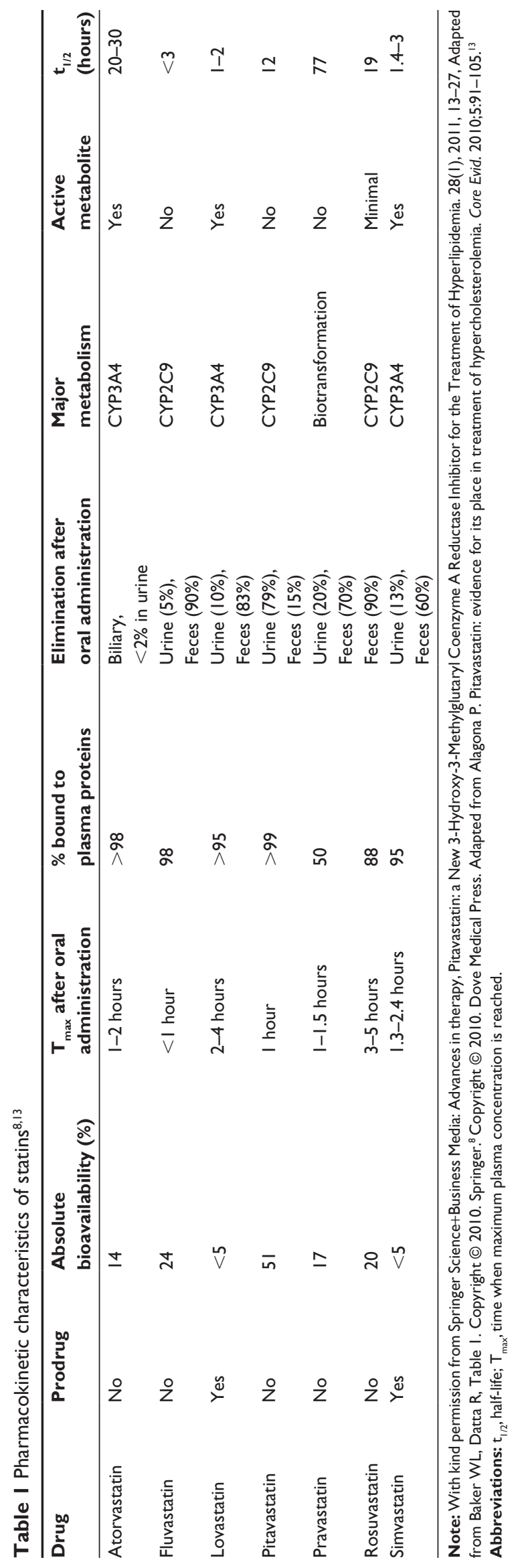


Pitavastatin is rapidly metabolized primarily via hepatic glucuronidation with UDP glucuronyltransferase (UGT1A3 and UGT2B7), forming the major inactive metabolite pitavastatin lactone - with minimal CYP2C9 and $2 \mathrm{C} 8$ metabolism of clinical concern. ${ }^{13}$ Pitavastatin is rapidly taken up into the liver through OATP, including OATP1B1 and OATP2. ${ }^{14,15}$ The 24-hour AUC of pitavastatin was increased 4.6-fold when it was concurrently administered with a potent OATP2 inhibitor (eg, cyclosporine), and is thought to be the major rate-limiting step in the hepatobiliary clearance of pitavastatin. ${ }^{16}$ The plasma elimination half-life of pitavastatin is between 11 and 12 hours, with approximately $15 \%$ of the dose excreted in the urine, and a mean of $79 \%$ of the dose excreted in the feces within 7 days. ${ }^{7,13}$

To date, pharmacokinetic studies have not demonstrated clinically significant differences based either on sex (men versus [vs] women) or age (younger vs elderly, age $>65$ years). Morgan et al studied the effects of severe renal impairment not on hemodialysis on pitavastatin metabolism, transport, and clearance. ${ }^{17}$ The study looked at age, body mass index, and sex-matched healthy adult subjects, using an US Food and Drug Administration (FDA)-approved cut-off of "healthy" estimated GFR of $\geq 80 \mathrm{~mL} / \mathrm{min} / 1.73 \mathrm{~m}^{2}$ to align the study with a previous pitavastatin analysis conducted in Europe. ${ }^{17}$ Elevations in AUC and $\mathrm{C}_{\max }$ were not found to be significantly different from their healthy comparators. They concluded that the relationship between renal function and pitavastatin exposure may not be evident, partly due to limited urinary excretion, though they admitted that there was a small sample size $(\mathrm{n}=16)$ and single dosing $(4 \mathrm{mg})$ in determining that pitavastatin was safe and well tolerated in patients with severe renal impairment not on hemodialysis. ${ }^{17}$

Pharmacokinetic studies have not demonstrated clinically significant differences based either on age (younger vs elderly - age $>65$ years) or sex (men vs women). ${ }^{7}$ Significant increases in $\mathrm{C}_{\max }$ have been seen in cirrhotic patients when compared with healthy subjects. ${ }^{18}$ Similarly, the elimination half-life was 8.3 hours and 14.4 hours in patients with ChildPugh A and Child-Pugh B classes, respectively.

\section{Efficacy studies of pitavastatin}

Information on the lipid-lowering efficacy of pitavastatin in elderly patients comes either from subgroup analysis of age-diverse studies ${ }^{19,20}$ or those performed specifically in older adults. ${ }^{21-23}$ A Phase 3, randomized, double-blind, parallel-group, active-controlled study of 355 patients by Eriksson et al compared pitavastatin ( $4 \mathrm{mg} /$ day) to simvastatin
(40 mg/day) over a 12-week period. ${ }^{19}$ Patients $\geq 65$ years of age represented $23 \%$ of the population. While no significant difference in percent lowering of LDL-C from baseline was seen between pitavastatin $(-44 \%)$ and simvastatin $(-42 \%)$ in those $<65$ years, a greater reduction was shown with simvasta$\operatorname{tin}(-48 \%)$ vs pitavastatin $(-43 \% ; P=0.024)$ in those $\geq 65$ years of age although the overall reduction between age groups was similar. ${ }^{19}$ A recently published study by Sponseller et al randomized 328 subjects with primary hyperlipidemia or mixed (combined) dyslipidemia to either pitavastatin $4 \mathrm{mg} /$ day or pravastatin $40 \mathrm{mg}$ /day for 12 weeks. $^{20}$ The reduction in LDL-C from baseline in the overall population was $-38.1 \%$ with pitavastatin and $-26.4 \%$ with pravastatin $(P<0.001)$. In subjects $<65$ years of age, the median percent change in LDL-C from baseline was $-36.3 \%$ with pitavastatin and $-25.5 \%$ with pravastatin while the reduction in those $\geq 65$ years of age was $-41.9 \%$ with pitavastatin and $-27.6 \%$ for pravastatin (Figure 1). The exact rationale for the greater response in the older population is unknown. Whether differences in baseline LDL-C concentrations could have resulted in varying reductions is also not clear. The magnitude of within-treatment effect for pravastatin was similar between those $<$ or $\geq 65$ years of age, the age effect on reduction in LDL-C was greater for pitavastatin vs simvastatin $\left(P_{\text {interaction }}=0.02\right) .{ }^{20}$

The efficacy of pitavastatin exclusively in a population $\geq 65$ years of age was evaluated by Stender et al in 942 patients with primary hypercholesterolemia or combined (mixed) dyslipidemia. ${ }^{21}$ Participants, who had a mean age of 70 years (range 65-89), were randomized to receive either pitavastatin $(1,2$, or $4 \mathrm{mg} /$ day $)$ or pravastatin $(10,20$, or 40 $\mathrm{mg} /$ day) for 12 weeks in a double-blind, double-dummy fashion. The primary efficacy outcome was the percent change in LDL-C from baseline with analyses performed to demonstrate the noninferiority of pitavastatin versus pravastatin. Additional lipoprotein parameters of interest included changes in TC, HDL-C, TG, and Apo-B. The following dosage comparisons were made: pitavastatin $1 \mathrm{mg}$ vs pravastatin $10 \mathrm{mg}$; pitavastatin $2 \mathrm{mg}$ vs pravastatin $20 \mathrm{mg}$; and pitavastatin $4 \mathrm{mg}$ vs pravastatin $40 \mathrm{mg}$. The mean baseline LDL-C across the various dosing groups ranged from $163 \mathrm{mg} / \mathrm{dL}$ to $167 \mathrm{mg} / \mathrm{dL}$. The mean decrease in LDL-C from baseline decreased across all doses in both arms in a dose-related fashion (Figure 2). The percent LDL-C reduction was approximately $10 \%$ greater with pitavastatin versus pravastatin, which met the criteria for noninferiority $(P<0.001)$. Pitavastatin nearly uniformly improved secondary outcome parameters vs pravastatin, including TC, HDL-C (except pitavastatin $1 \mathrm{mg}$ vs pravastatin $10 \mathrm{mg}$; 


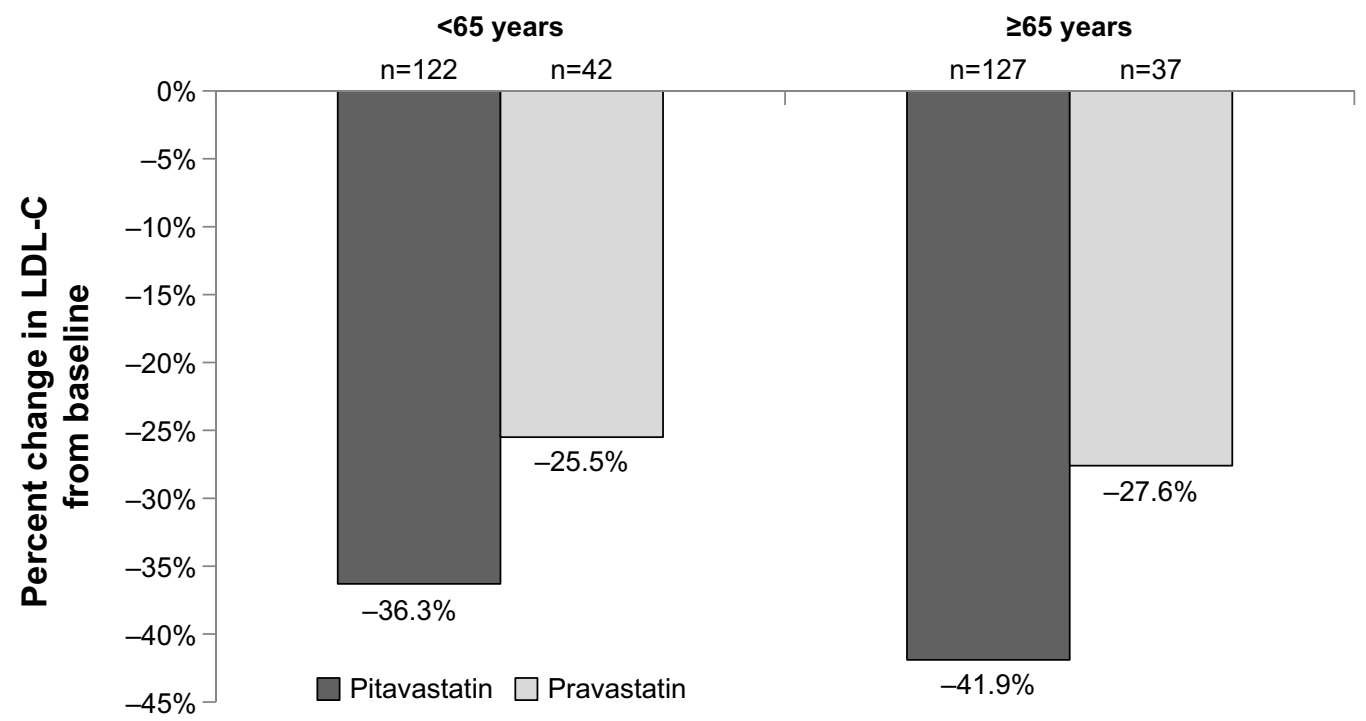

Figure I Percent LDL-C lowering ability of pitavastatin versus pravastatin by age.

Notes: $P_{\text {interaction }}=0.02$ for effect of age on \% LDL-C reduction. Data from Sponseller et al. ${ }^{20}$

Abbreviation: LDL-C, low-density lipoprotein cholesterol.

$P=0.425)$, TG (except pitavastatin $2 \mathrm{mg}$ vs pravastatin $20 \mathrm{mg} ; P=0.063)$, Apo-B, and non-HDL-C $(P<0.05$ for all, except where specified).

The same author group concurrently published results of an open-label, 60-week extension study for those who had completed the 12-week core study. ${ }^{22}$ All patients, irrespective of their original randomization group, were started on openlabel pitavastatin $2 \mathrm{mg} /$ day, then up-titrated to $4 \mathrm{mg}$ /day after 8 weeks if they did not attain their LDL-C target. The specific LDL-C target was not provided, but was based on the individual patients' risk and followed the National Cholesterol Education Program Adult Treatment Panel III
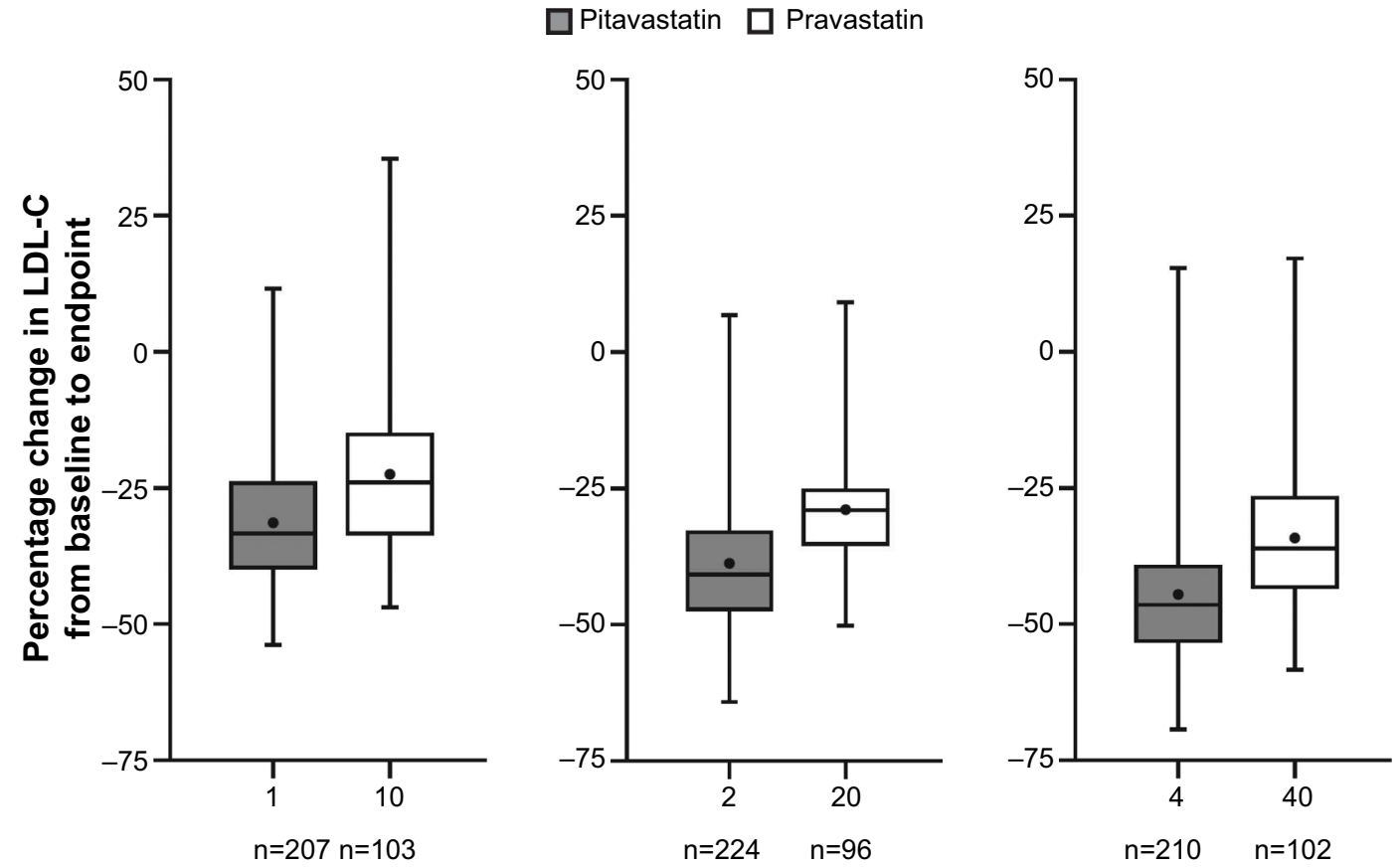

Dose (mg/day)

Figure 2 Change in LDL-C with pitavastatin versus pravastatin in patients aged $\geq 65$ years.

Notes: Stender S, Budinski D, Gosho M, Hounslow N. Pitavastatin shows greater lipid-lowering efficacy over 12 weeks than pravastatin in elderly patients with primary hypercholesterolemia or combined (mixed) dyslipidemia. Eur J Prev Cardiol. 20(I):40-53, copyright @ 20I3 by (SAGE Publications). Reprinted by Permission of SAGE. ${ }^{21}$ Abbreviation: LDL-C, low-density lipoprotein cholesterol. 
guidelines. Following 60 weeks of pitavastatin treatment, the LDL-C was $43 \%$ lower than at baseline of the 12 -week core study. ${ }^{21}$ All other secondary lipid parameters were also lower after 60 weeks compared with baseline. While $85.8 \%$ and $72.9 \%$ of pitavastatin and pravastatin (respectively) patients had attained their LDL-C goals after the 12-week core study, ${ }^{21}$ the value increased to $93.8 \%$ after 60 weeks of pitavastatin. ${ }^{22}$

Comparative efficacy information for pitavastatin as well as other statins in patients $<65$ years and $\geq 65$ years of age was included when the agent was submitted for approval to the FDA. ${ }^{23}$ The percent LDL-C reductions in LDL-C for patients in the $\geq 65$ versus $<65$ years groups are shown in Figure 3 . $^{23}$ It should be noted that direct statistical comparisons between these groups were not conducted, nor inferred.

Information on the non-lipid characteristics of pitavastatin in elderly patients is also available. The effect of pitavastatin on prevention of atrial fibrillation as well as cardiac structure and function was evaluated in 220 patients $\geq 65$ years of age. ${ }^{24}$ Individuals with hypertension and established leftventricular (LV) hypertrophy and preserved systolic function ( $\mathrm{LV}$ ejection fraction $\geq 50 \%$ ) were included. Patients who required lipid lowering therapy $(n=110)$ according to Japanese guidelines were started on pitavastatin (1-2 mg/day) in addition to antihypertensive treatment, while the control group $(n=110)$ continued on their baseline antihypertensive regimens. Treatment and follow-up was continued for a 1-year period. All baseline clinical, laboratory, and echocardiographic parameters were similar between the two groups, with the exception of LDL-C. Patients receiving pitavastatin had a significantly lower rate of developing new-onset atrial fibrillation $(5 / 110,4.5 \%)$ than the non-statin group $(15 / 110$, $13.6 \% ; P=0.019)$. In a multivariate Cox regression model, pitavastatin use $(P=0.002)$ and an echocardiographic finding (left atrial peak strain; $P=0.013$ ) were independent predictors of reduced atrial fibrillation risk while prior coronary artery disease $(P<0.001)$ was associated with elevated risk. Moreover, pitavastatin use resulted in a $6 \%$ reduction in $\mathrm{LV}$ mass index from baseline with no difference seen in the nonstatin group $(P<0.05)$. This study shows that pitavastatin not only reduces lipid parameters, but also improves echocardiographic parameters in patients with hypertension and LV hypertrophy and lowers their risk of developing new-onset atrial fibrillation.

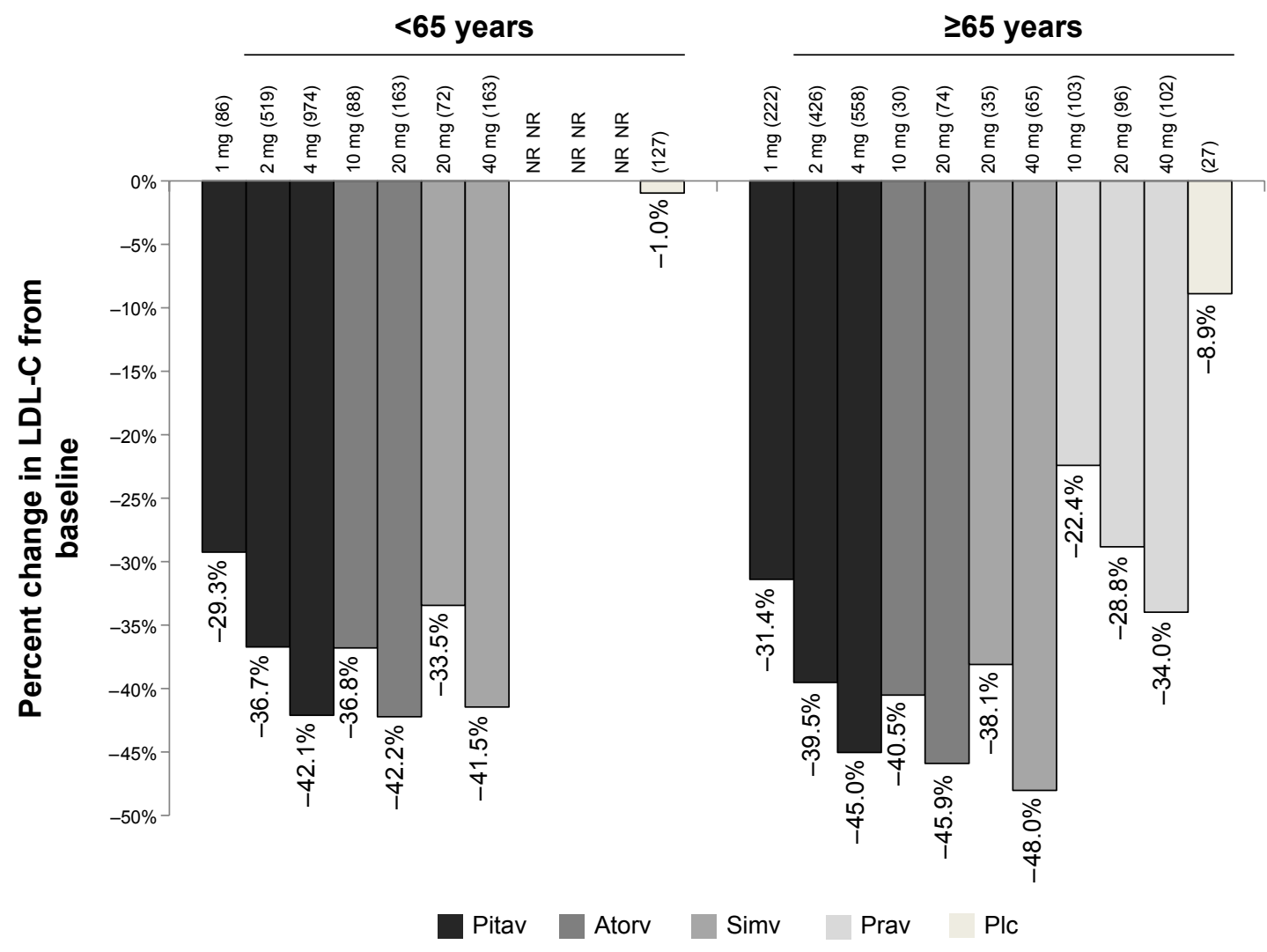

Figure 3 Mean percent LDL-C lowering ability of statins by age.

Notes: Patient numbers are shown in parentheses. Data from US Food and Drug Administration. Clinical Review Pitavastatin. Center for Drug Evaluation and Research; 2009. Available from: http://www.accessdata.fda.gov/drugsatfda_docs/nda/2009/022363s000_MedR_PI.pdf. ${ }^{23}$

Abbreviations: Atorv, atorvastatin; Pitav, pitavastatin; Plc, placebo; Prav, pravastatin; Simv, simvastatin; LDL-C, low-density lipoprotein cholesterol; NR, not reported. 


\section{Safety and tolerability of pitavastatin in elderly patients}

The manufacturer reports that of the 2,800 patients randomized to pitavastatin $1 \mathrm{mg}$ to $4 \mathrm{mg}$ in controlled clinical studies, 1,209 (43\%) were $\geq 65$ years of age. No significant differences in efficacy or safety were observed between elderly patients and younger patients when pitavastatin was approved by the FDA. ${ }^{7}$

Stender et al reported that $82 \%$ of pitavastatin patients in their 60-week open-label extension trial developed adverse events; however, only $14.3 \%$ of these were deemed to be specifically related to drug treatment. ${ }^{22}$ The most common treatment-related adverse events included myalgia (2.6\%), elevated serum creatine kinase (1.9\%), and nausea (1.5\%). Fewer than $7 \%$ of patients discontinued pitavastatin therapy because of the adverse events. Two patients died during the study, although neither death was attributed to pitavastatin therapy.

Stender et al reported 48 cases of myalgias; however, only 14 of these were considered treatment related, and none were severe ( 21 were moderate, 27 were mild). ${ }^{22}$ Only four patients discontinued pitavastatin due to myalgia, and no cases of myopathy, myositis, or rhabdomyolysis were identified.

Patients receiving pitavastatin $4 \mathrm{mg} /$ day had a higher incidence of elevations in liver enzymes (AST and ALT) by week $60 .{ }^{22}$ One patient had an AST elevation three times the upper limit of normal (ULN), and one more than five times the ULN. Two patients had ALT increases more than three times the ULN, one more than five, and one more than ten. Two patients were said to have discontinued the study due to increases in ALT and AST, both of which were assessed as mild intensity and only one identified as treatment-related. Taken together, despite elevations in liver function tests (LFTs) in a handful of patients, significant liver injury requiring treatment discontinuation was rare.

Other studies have included elderly patients in their assessments of pitavastatin safety and tolerability; however, limited subgroup analysis data exist specific to those patients aged 65 years and older. ${ }^{25-27}$ The significant majority of adverse events were reported as mild and less frequent than with other potent statins, and compare similarly to the data from Stender et al. ${ }^{22}$

Older patients who have been deemed frail may be at increased risk for adverse events. ${ }^{1}$ This includes individuals with a combination of weakness, slowness, exhaustion, inactivity, and shrinking. ${ }^{1}$ Unfortunately, data on the safety of pitavastatin in frail older patients are lacking and requires additional study.

\section{Patient focused perspectives (quality of life, patient satisfaction, and acceptability/adherence)}

Information on the impact of pitavastatin on humanistic outcomes, such as health-related quality of life, patient satisfaction, and treatment adherence is limited. No data on the impact of pitavastatin on health-related quality of life could be found. Patient satisfaction was evaluated in a Spanish study of 6,489 patients from primary or specialized health clinics in Spain who had been using pitavastatin for at least 12 weeks. ${ }^{28}$ The mean age of the population was $60.9 \pm$ 11.2 years, with no age-related subgroup data provided. Patients who completed their prescribed treatment course (65\% of the included population) showed improved satisfaction with the drug and with the drug efficacy than those who did not finish the treatment ( $P<0.001$ for both). Treatment compliance in elderly patients was reported in an extension study by Stender et al. ${ }^{22}$ Compliance, defined as taking between $80 \%-120 \%$ of the prescribed treatment regimen, was achieved in $97.5 \%$ of the 60 -month follow-up population.

\section{Conclusions, benefit-risk assessment}

The overall body of evidence for the efficacy and safety of pitavastatin in elderly patients is small. The available data suggest that the ability of pitavastatin to lower LDL-C in elderly patients is at least similar, and may be greater than that seen in comparatively younger cohorts. A similar trend was seen with other statins, including atorvastatin, pravastatin, and simvastatin. Percent reductions ranging from $31 \%-45 \%$ were seen with pitavastatin compared with $22 \%-34 \%$ with pravastatin, $38 \%-48 \%$ with simvastatin, and $40 \%-46 \%$ with atorvastatin in patients $\geq 65$ years of age. ${ }^{23}$ Studies using rosuvastatin $10 \mathrm{mg} /$ day in patients $\geq 65$ years of age have shown $50 \%$ reductions in LCL-C from baseline, proportionally greater than pitavastatin. ${ }^{29}$ Real-world studies of elderly patients have shown rosuvastatin to result in greater LDL-C reductions from baseline compared with other drugs in the class, including atorvastatin, pravastatin, and simvastatin. ${ }^{30}$ Pitavastatin was not available at the time of this investigation; thus, the comparative efficacy of it with rosuvastatin is not currently known.

Clinical trials have shown that statins significantly reduce all-cause mortality, nonfatal myocardial infarction, and stroke in elderly patients. ${ }^{4}$ The Study Assessing Goals in the Elderly (SAGE) of patients 66-85 years of age showed that more aggressive lipid lowering with atorvastatin reduced cholesterol and mortality to a greater extent than moderate intensity therapy with pravastatin. ${ }^{31}$ Unfortunately, data on the impact of pitavastatin on terminal outcomes such as mortality and 
myocardial infarction are not available. Pitavastatin has been shown to have similar pleiotropic effects to other statins, including decreasing platelet activation and improving cardiac and endothelial function. ${ }^{32}$ Thus, it is plausible that its use may confer similar clinical benefits, but these have yet to be elucidated. It should, therefore, be selected based on its lipid lowering ability and safety profile alone.

The safety of statins in elderly patients is a question frequently encountered by clinicians and patients alike. One of the biggest concerns, from a patient perspective, is the risk of statins adversely affecting cognition. A systematic review suggested that no causal link existed between statin use and development of Alzheimer disease or dementia. ${ }^{33}$ Similarly, no decline in cognitive performance was seen with statin use. A task force commissioned by the National Lipid Association concluded that no association with the statin class and adverse effects on cognition are known and routine screening should not be performed. ${ }^{34}$ While no information on the specific effects of pitavastatin on cognition in elderly patients is currently available, this adverse effect should not be of concern.

Muscle issues have also been a concern with statins. ${ }^{35}$ Meta-analyses of observational studies in general populations have reported greater than a two-fold increase in the odds of myopathy with statin use (odds ratio $2.63,95 \%$ confidence interval 1.50 to 4.61$).{ }^{35}$ While statin-associated myalgia may be difficult to differentiate from that caused by other sources, tests can be run on individuals who have exhibited symptoms to provide a definitive diagnosis. ${ }^{36}$ Stender et al reported that myalgia $(2.6 \%)$ and increased serum creatine kinase $(1.9 \%)$ did occur in elderly patients receiving pitavastatin. ${ }^{22}$ This is compared with an approximate $3 \%$ incidence with moderately-potent comparators. ${ }^{23}$

Liver toxicity is another safety issue routinely encountered with statin use. ${ }^{35}$ Few of the elderly patients given pitavastatin by Stender et al exhibited signs or symptoms suggestive of liver damage. ${ }^{22}$ Elevations in LFTs are seen at a similar rate between pitavastatin and other agents in the class. ${ }^{23}$ While LFTs are not recommended to be routinely screened following statin initiation, they can be checked in individuals with symptoms suggestive of liver damage. ${ }^{37}$

Taken together, the limited available data suggest that pitavastatin is effective at improving lipid parameters in elderly patients with a similar safety profile to other agents in the class. Current guidelines recommend its use when moderate-intensity statin therapy is indicated. ${ }^{6}$ A drawback to its use is likely to be its cost. Since pitavastatin is still available only as a branded product in the United States, other agents with similar efficacy and safety and lower cost are likely to be used with greater frequency. Until data become available distinguishing pitavastatin from the other available options, its ultimate role in the hyperlipidemia treatment armamentarium remains unclear.

\section{Disclosure}

The authors declare no relevant conflicts of interest.

\section{References}

1. Strandberg TE, Kolehmainen L, Vuorio A. Evaluation and treatment of older patients with hypercholesterolemia: a clinical review. JAMA. 2014;312(11):1136-1144.

2. Prospective Studies Collaboration; Lewington S, Whitlock G, et al. Blood cholesterol and vascular mortality by age, sex, and blood pressure: a meta-analysis of individual data from 61 prospective studies with 55,000 vascular deaths. Lancet. 2007;370(9602):1829-1839.

3. Schatz IJ, Masaki K, Yano K, et al. Cholesterol and all-cause mortality in elderly people from the Honolulu Heart Program: a cohort study. Lancet. 2001;358(9279):351-355.

4. Afilalo J, Duque G, Steele R, et al. Statins for secondary prevention in elderly patients: a hierarchical Bayesian meta-analysis. $J$ Am Coll Cardiol. 2008;51(1):37-45.

5. Stone NJ, Robinson JG, Lichtenstein AH, et al. 2013 ACC/AHA guideline on the treatment of blood cholesterol to reduce atherosclerotic cardiovascular risks in adults: a report of the American College of Cardiology/American Heart Association Task Force on Practice Guidelines. Circulation. 2014;129(25 Suppl 2):S1-S45.

6. Clegg A, Young J, Lliffe S, et al. Frailty in elderly people. Lancet. 2013; 381(9868):752-762.

7. Livalo ${ }^{\circledR}$ (pitavastatin) [package insert]. Montgomery, AL: Kowa Pharmaceuticals America, Inc.; 2013.

8. Baker WL, Datta R. Pitavastatin: a New 3-Hydroxy-3-Methylglutaryl Coenzyme A Reductase Inhibitor for the Treatment of Hyperlipidemia. Adv Ther. 2011;28(1):13-27.

9. Aoki T, Nishimura H, Nagakawa S, et al. Pharmacologic profile of a novel synthetic inhibitor of 3-hydroxy-3-methylglutaryl-coenzyme A reductase. Arzneimittelforschung. 1997;47(8):904-909.

10. Morikawa S, Umetani M, Nakagawa S, et al. Relative induction of mRNA for HMG CoA reductase and LDL receptor by five different HMGCoA reductase inhibitors in cultured human cells. J Atheroscler Thromb. 2000;7(3):138-144.

11. Suzuki H, Aoki T, Tamaki T, et al. Hypolipidemic effect of NK-104, a potent $\mathrm{HMG}-\mathrm{CoA}$ reductase inhibitor, in guinea pigs. Atherosclerosis. 1999; 146(2):259-270.

12. Saito Y. Critical appraisal of the role of pitavastatin in treating dyslipidemias and achieving lipid goals. Vasc Health Risk Manag. 2009;5: 921-936.

13. Alagona P. Pitavastatin: evidence for its place in treatment of hypercholesterolemia. Core Evid. 2010;5:91-105.

14. Shimada S, Fujino H, Hojima J, Moriyasu M, Kojima J. Uptake mechanism of pitavastatin, a new inhibitor of HMG-CoA reductase, in rat hepatocytes. Drug Metab Pharmacokinet. 2003;18(4):245-251.

15. Hirano M, Maeda K, Shitara Y, Sugiyama Y. Contribution of OATP2 (OATP1B1) and OATP8 (OATP1B3) to the hepatic uptake of pitavastatin in humans. J Pharmacol Exp Ther. 2004;311(1):139-146.

16. Hasanuma T, Nakamura M, Yaji T, et al. The drug-drug interactions of pitavastatin (NK-104), a novel HMG-CoA reductase inhibitor and cyclosporine. J Clin Therap Med. 2003;19(4):381-389.

17. Morgan RE, Campbell SE, Yu CY, Sponseller CA, Muster HA. Comparison of the safety, tolerability, and pharmacokinetic profile of a single oral dose of pitavastatin $4 \mathrm{mg}$ in adult subjects with severe renal impairment not on hemodialysis versus healthy adult subjects. J Cardiovasc Pharmacol. 2012;60(1):42-48. 
18. Hui CK, Cheung MY, Lau GK. Pharmacokinetics of pitavastatin in subjects with Child-Pugh A and B cirrhosis. Br J Clin Pharmacol. 2004;59(3):291-297.

19. Eriksson M, Budinski D, Hounslow N. Comparative efficacy of pitavastatin and simvastatin in high-risk patients: a randomized controlled trial. Adv Ther. 2011;28(9):811-823.

20. Sponseller CA, Morgan RE, Kryzhanovski VA, Campbell SE, Davidson MH. Comparison of the lipid-lowering effects of pitavastatin $4 \mathrm{mg}$ versus pravastatin $40 \mathrm{mg}$ in adults with primary hyperlipidemia or mixed (combined) dyslipidemia: a phase IV, prospective, US, multicenter, randomized, double-blind, superiority trial. Clin Ther. 2014;36(8):1211-1222.

21. Stender S, Budinski D, Gosho M, Hounslow N. Pitavastatin shows greater lipid-lowering efficacy over 12 weeks than pravastatin in elderly patients with primary hypercholesterolemia or combined (mixed) dyslipidemia. Eur J Prev Cardiol. 2013;20(1):40-53.

22. Stender S, Budinski D, Hounslow N. Pitavastatin demonstrates longterm efficacy, safety and tolerability in elderly patients with primary hypercholesterolemia or combined (mixed) dyslipidaemia. Eur J Prev Cardiol. 2013;20(1):29-39.

23. US Food and Drug Administration. Clinical Review Pitavastatin. Center for Drug Evaluation and Research; 2009. Available from: http://www. accessdata.fda.gov/drugsatfda_docs/nda/2009/022363s000_MedR_ P1.pdf. Accessed January 29, 2015.

24. Warita S, Kawasaki M, Tanaka R, et al. Effect of pitavastatin on cardiac structure and function and on prevention of atrial fibrillation in elderly hypertensive patients. A prospective study of 2-years' follow-up. Circ J. 2012;76(12):2755-2762.

25. Ose L, Budinski D, Hounslow N, Arneson V. Comparison of pitavastatin with simvastatin in primary hypercholesterolaemia or combined dyslipidaemia. Curr Med Res Opin. 2009;25(11):2755-2765.

26. Ose L, Budinski D, Hounslow N, Arneson V. Long-term treatment with pitavastatin is effective and well tolerated by patients with primary hypercholesterolemia or combined dyslipidemia. Atherosclerosis. 2010;210(1):202-208

27. Gumprecht J, Gosho M, Budinski D, Hounslow N. Comparative long-term efficacy and tolerability of pitavastatin $4 \mathrm{mg}$ and atorvastatin $20-40 \mathrm{mg}$ in patients with type 2 diabetes mellitus and combined (mixed) dyslipidaemia. Diabetes Obes Metab. 2011;13(1):1047-1055.
28. Rodriquez Arroyo LA, Diaz Rodriquez A, Pinto Sata X, Coca Paveras A, Rius Taruella J. [Effectivity and satisfaction with the treatment of dyslipidemia with pitavastatin. Multicentric, descriptive, post authorized and observational study (REINA study)]. Clin Investig Arterioscler. 2014;26(5):205-217. Spanish.

29. Blasetto JW, Stein EA, Brown WV, Chitra R, Raza A. Efficacy of rosuvastatin compared with other statins at selected starting doses in hypercholesterolemic patients and in special population groups. $\mathrm{Am}$ J Cardiol. 2003;91(5A):3C-10C.

30. Harley CR, Gandhi S, Blasetto J, et al. Low-density lipoprotein cholesterol (LDL-C) levels and LDL-C goal attainment among elderly patients treated with rosuvastatin compared with other statins in routine clinical practice. Am J Geriatr Pharmacother. 2007;5(3):185-194.

31. Deedwania P, Stone PH, Bairey Merz CN, et al. Effects of intensive versus moderate lipid-lowering therapy on myocardial ischemia in older patients with coronary heart disease: results of the Study Assessing Goals in the Elderly (SAGE). Circulation. 2007;115(6):700-707.

32. Davignon J. Pleiotropic effects of pitavastatin. Br J Clin Pharmacol. 2011;73(4):S18-S35.

33. Richardon K, Schoen M, French B, et al. Statins and cognitive function: a systematic review. Ann Intern Med. 2013;159(10):688-697.

34. Rojas-Fernandez CH, Goldstein LB, Levey AI, Taylor BA, Bittner V; The National Lipid Association's Safety Task Force. An assessment by the Statin Cognitive Safety Task Force: 2014 update. J Clin Lipidol. 2014;8(3 Suppl):S5-S16.

35. Macedo AF, Taylor FC, Casas JP, Adler A, Prieto-Merino D, Ebrahim S. Unintended effects of statins from observational studies in the general population: systematic review and meta-analysis. $B M C$ Med. 2014;22:51.

36. Rosensen RS, Baker SK, Jacobson TA, Kopecky SL, Parker BA; The National Lipid Association's Muscle Safety Expert Panel. An assessment by the Statin Muscle Safety Task Force: 2014 update. J Clin Lipidol. 2014;8(3 Suppl):S58-S71.

37. Bays H, Cohen DE, Chalasani N, Harrison SA; The National Lipid Association's Statin Safety Task Force. An assessment by the Statin Liver Safety Task Force: 2014 update. J Clin Lipidol. 2014; 8(3 Suppl):S47-S57.
Clinical Interventions in Aging

\section{Publish your work in this journal}

Clinical Interventions in Aging is an international, peer-reviewed journal focusing on evidence-based reports on the value or lack thereof of treatments intended to prevent or delay the onset of maladaptive correlates of aging in human beings. This journal is indexed on PubMed Central, MedLine,

\section{Dovepress}

CAS, Scopus and the Elsevier Bibliographic databases. The manuscript management system is completely online and includes a very quick and fair peer-review system, which is all easy to use. Visit http://www.dovepress. com/testimonials.php to read real quotes from published authors. 